\title{
Annotating Educational Content by Questions Created by Learners
}

\author{
Maroš Unčík and Mária Bieliková \\ Institution of Informatics and Software Engineering \\ Faculty of Informatics and Information Technologies, Slovak University of Technology \\ Ilkovičova 3, 84216 Bratislava, Slovakia \\ xuncik@stuba.sk,maria.bielikova@stuba.sk
}

\begin{abstract}
Several approaches to educational web-based content enrichment have been devised. Annotations in form of comments and other types of remarks obviously supply these approaches. Annotations allow enriching the educational materials mainly by retaining key information or comments; they can support visual search and also collaboration. In this paper we present a method for an acquisition of new educational content created by learners. It is based on collaborative questions creation. We let the students to enrich educational materials with questions related to the selected text. We believe that this helps them to learn more effectively. It facilitates a collaboration and explicit rating to gain new quality content. This kind of annotations can be considered also as a source of semantics of the selected text and used for adaptation. To evaluate proposed method we experimented in domain of learning programming. We implemented the method within an existing web-based educational framework ALEF and provided several experiments.
\end{abstract}

\section{Introduction}

Using the Web as an infrastructure for education originated a need of approaches for providing effective presentation of educational materials to learners. Nowadays, many educational web-based systems provide not just static content, but also provide an adaptation to the individual needs of a student or a group of students and support learning also by communication and advice. Important role in this situation plays the actual content. It can exist in several forms - from educational text together with media through various discussions to annotations. Annotations present the common way of the content enrichment [7]. Annotations provide a way to discussion and supply context based communication of students and their teachers. Students add annotations into the educational materials, which can lead to a discussion among them and the teacher.

The classical educational environment consists of passive consumers (learners, students) and creators (teachers) of the educational content. Our idea is to integrate passive parts of the learning process and make them active. Idea of employing the students as the content creators has been used in the several studies [5]. There are several advantages of this approach. Employing students as creators increases their understanding of educational texts and provides collaborative learning opportunity. The students are not just studying, but they also create the content which is useful for their peers, which can be motivational. The most important advantage of this activity is that students actively and directly use their knowledge in creating new content, which can serve for the assessment of their level of knowledge. It also provides a way to communication. If we allow students to insert a new content, it leads to an information exchange. The other advantage is gaining new content thanks to the collaboration. However, we should consider and estimate quality of such content to be used for learning.

The potential advantages of annotations are relevant in the context of web-based e-learning, where users are students and teachers. On one hand, students browse and annotate an educational text in order to understand it, and summarize the key information. They may want to share their own annotations with others students or teachers to collaboratively solve problems, ask for help, or to clear up some concepts. On the other hand, teachers may annotate and highlight related information, post announcements, or address some important comments raised by students.

In this paper we present a method for educational content enrichment. We proposed an approach for adding new and interactive content to educational materials and evaluate added content by estimating its quality. We propose the adding questions based on 
selected part of the document. The questions are added by students, who also participate on answering and reviewing questions created by their peers.

Questions present important element of any educational material. Even though, there exist some approaches to automatic question extracting, the quality of extracted questions is still low. On the other hand, creating questions by an expert is extremely time-consuming and from the expert view it is often difficult to specify the difficulty level of questions.

We follow the idea raised in our previous work on considering questions as a part of the educational content $[1,12]$. We achieved promising results in incorporating questions and exercises within educational material to facilitate learning (not just putting them at the end of session or chapter to review student's knowledge, but use it for actual learning). We propose to move this further and conceptualize questions as annotations. Our questions are tasks created by students and are intended to their peers. The question as an annotation concerns selected part of the content similarly to the annotation. The process of such questions creation is almost identical to the process of making annotations [7].

\section{Related Work}

Web content annotation is an area of research, which has been active since genesis of the Web. By annotations, in the context of this paper we mean various remarks that are related to the document educational text or web page. Many web-based systems support various types of annotations. Underlining and highlighting are common techniques widely used in educational web-based systems [4].

One of the first content annotation systems have already appeared in 1993. Knowledge Weasel Hypermedia Annotation System [6] allows annotating various sources. It maintains a consistent and easy access to all annotations and their presentation through one interface. This principle is used in many approaches today. Current annotation systems partly realize automatic annotation and at the same time allow a user-added content. Using techniques for information extraction and semantics discovery is the common way of automatic annotation approaches, which are based on a mapping of extracted information (metadata) to the document based on its semantics [8]. The user content enrichment is different as new content is added by the users' logic, which cannot be managed, just directed. A number of such web-based annotation tools for educational environment already exist $[2,3]$.

An example of manual annotation system is a browser plug-in Diigo (www.diigo.com). It allows commenting of any web page, using two types of notes. Highlighting text or any content on a web page and adding "sticky notes" are connected together. The system allows commenting notes by other users which open communication space for users. Google SideWiki allows adding further information to any web page (www.google.com/sidewiki/). Another example is the Sidenote (www.sidenote.com), which is a graphical tool for commenting the content on the Web. It allows adding graphical notes by drawing to the additional layer above the document. The major drawback of this system is lack of sharing notes between users; they cannot see comments neither response to the notes of their colleagues.

Annotating documents in a web-based educational environment is based on similar principles. However, the adding of new content in educational context is important to evaluate from the content quality point of view. Manual content enrichment by students' collaboration can be improved by form of mutual review and control [5].

Another approach to manual annotation uses A.nnotate (www.a.nnotate.com). It focuses on annotating documents, which are accessible through a browser. A user can add annotations and select regions in the document and impress a standalone application.

Most current annotation systems are realized as automatic annotating engines for web pages, educational materials or for the user commenting only. The systems in most cases enable collaboration in various forms. The common meaning of annotations in such systems is a statement about the document or its part. Annotations in this form are often considered also as static metadata, which usually do not add new facts or new content. They mostly only summarize characteristics of document.

\section{Annotation Process}

Most important prerequisite of an automatic annotation is the existence of an annotation base. Annotations are selected from the base according to defined rules and visualized at suitable location in the document. Such requirement is not necessary for manual annotation where a user is the source of annotations. We consider such manual process where annotations are added directly by users. It can be divided into three main steps (i) studying the document, (ii) deciding what to insert as an annotation, and where the annotation insert, (iii) providing the annotation.

We emphasize other step - the collaboration. It has several aspects. First, we regard such collaboration as the cooperation of users (learners) for information exchange. Another aspect of collaboration is creating annotations based on the feedback from peers. Such 
feedback is reflected in an explicit rating of the quality of comments based on its relevance or other characteristics. Another significant aspect of the collaboration is the user feedback for the author of the document (teacher) in form of opinions, observations or highlighting of the errors.

Collaborative annotations are particularly suitable for the educational domain. Here, annotations create a space for discussion, thus, students and teachers can effectively communicate and share their knowledge. Their opinions and insights can be expressed directly to the point of the document to which they relate.

Annotating does not evoke the change of original document, data of the annotations and data of the document are separated, i.e., the annotating adds another dimension of the semantics to the document. Because the annotations represent added information bound to a particular document fragment, we propose to realize questions with answers on particular document part using annotations concept.

\section{Method of Questions Acquisition}

Adding user annotations, i.e. questions in our case, is a complex process. As questions are added by students who are just studying particular topic, it is necessary to evaluate questions quality in order to use them for learning. Our proposed concept of collaborative adding questions consists of creating, adding and rating questions. Evaluating the quality of questions is based on the explicit feedback of students in conjunction with actions that students do within the educational system combined also with the evaluation of an expert (a teacher). Our approach also includes a competitive element of motivation of students in the form of gaining points by simple game.

The entire method consists of two steps, which can be provided also in parallel:

1. Student rating determined by student rating model,

2. Question rating determined by question quality rating model.

Collaborative annotation involves two basic activities:

1. Creating of a question - a student selects a text and thinks out a question related to this text. This includes a selection of type of question (e.g. single choice, multi choice, short answers, matching) and creating the question according its type.

2. Answering the question - a student selects a question from the list of questions when studying particular learning object and answers the question according its type.

As an output we process the ratings of all questions, which is used for further improvement of educational materials together with valued estimations of student's level of knowledge in particular domain. Creating and answering questions are short-term activities. The process of rating students and questions are long term processes as we need certain amount of students' activity in order to be able evaluate their performance.

We also influence the students, so they are positive forced to achieve the best results to gain knowledge and also to create quality content by implementing ranging of students activity which is always visible.

The newly added question is persisted and it is bound to educational materials. These questions are displayed together with previously created questions related to the particular learning object (see Fig. 1 in section 5). The student can select questions for answering (the system also offers the next question option). When he chooses a question, the appropriate form for answering is shown, according to the type of question. The student labels or fills the correct answer, confirms and the system evaluates the answer.

\subsection{Student rating model}

We rate the ability of a student to add questions and estimate quality of each question. Student rating is based on proposed student rating model. Our aim is to quantify ability level of the adding questions for particular student. Actions provided by the student determine the student rating model. We consider the following critical factors:

\section{Rating the question creation:}

$$
\mathrm{C}_{\mathrm{Qu}}=\frac{\log \left(\mathrm{c}_{\mathrm{Qu}}\right)}{\Delta \mathrm{t}_{\mathrm{c}}}
$$

where $u$ is a user, $c_{Q u}$ denotes the number of questions created by the user $u$ and $\Delta t_{c}$ denotes the difference between the last time of occurrence of this factor and the time of new occurrence.

\section{Rating the answering a question:}

$$
\mathrm{A}_{\mathrm{Qu}}=\frac{\log \left(\mathrm{a}_{\mathrm{Qu}}\right)}{\Delta \mathrm{t}_{\mathrm{a}}}
$$

where $a_{Q u}$ is the number of questions answered by the user $u$ and $\Delta t_{a}$ denotes the difference between the last time of occurrence of the factor and the time of new occurrence.

\section{Explicit rating of the question:}

$$
\mathrm{R}_{\mathrm{Qu}}=\frac{\log \left(\mathrm{r}_{\mathrm{Qu}}\right)}{\Delta \mathrm{t}_{\mathrm{r}}}
$$

where $r_{Q u}$ is the number of questions rated by the user $u$ and $\Delta t_{r}$ denotes the difference between the last time of occurrence of the factor and time of new occurrence. 


\section{Similarity rating:}

$$
S_{u}=\prod_{i \in \tau} s_{u}\left(u_{u}, u_{i}\right)
$$

where $I$ is the set of all users, $s_{u}\left(u_{u}, u_{i}\right)$ is the cosine similarity between the user $u$ and the user $i$ represented by the rating vector $x$ and $y$ :

$$
s_{u}(x, y)=\frac{\sum x_{i} y_{i}}{\sqrt{\sum x_{i}^{2}} \sqrt{\sum y_{i}^{2}}}
$$

This factor represents a measure to which the user rates the questions like his peers. It is based on the idea of similarity of explicit rating by students [5]. Higher rated are the students who do not rate the questions extreme (by giving them too low, or too high rating).

Combining equations (1, 2, 3 and 4) we define the overall rating of the user $u$ :

$$
M_{u}=a C_{Q u}+b A_{Q u}+c R_{Q u}+d S_{u}
$$

The values of parameters $a, b, c$ and $d$ were determined by the experimentation with the model in particular context of usage (learning programming): $a=1.3, b=0.3, c=0.1$ and $d=0.5$. The values of parameters reflect the fact that the complex activities of the student earn more points than the easier ones and ensure that values are rising slowly.

\subsection{Question quality rating model}

Similarly to rating the ability of users to add questions, we rate the quality of questions. Question quality rating derives from the explicit question rating by the students and implicit question rating based on the actions of the students in the educational system.

The model of question quality rating is determined by four factors:

1. Explicit rating - arithmetic average of the explicit ratings of all students.

2. Count of right answers - normal distribution of the ratio of correct and wrong answers.

3. Count of "I don't understand" - calculated as the ratio of number of "I don't understand" labels and number of all answers to particular question. This factor reduces the question rating.

4. Count of mistakes - calculated as the ratio of the number of error labels on the question and the number of all answers of the question. This factor reduces the question rating.

Model of question rating is expressed by formula:

$$
r Q=\frac{\sum_{i=1}^{n}\left(M_{u i} e_{i}\right)}{n_{e}}+N\left(\frac{n_{T A}}{n_{A}}\right)-\operatorname{arctg}\left(\frac{n_{D K}}{n_{A}}\right)-\operatorname{arctg}\left(\frac{n_{M}}{n_{A}}\right)
$$

where $r_{Q}$ represents the rating of the question by the user $i, e_{i}$ denotes explicit rating given by the user $i$ for the question, $n_{T A}$ is the number of all correct answers for the question, $n_{A}$ is the number of all answers to the question. $n_{D K}$ is the number of answers to questions marked as "do not understand". $N$ denotes the function of normal distribution with parameters $\mu=0.5$ and $\delta^{2}$ $=0.2$, these values were determined experimentally so that the normal distribution has been defined in the domain of the function $<0, \infty)$ and range $<0,5>$.

The question rating is cumulative and is based on the current rating of the user. All parameters used in the model were determined experimentally. They can vary depending on actual needs, e.g., if the model is enhanced by a new rating factor.

As the final question quality rating depends on the set of parameters it is important to identify the boundaries in which we consider satisfactory question quality. In our settings - the quality questions were observed beyond value of 23.0. This limit threshold was determined to contain approximately $20 \%$ the best rated questions.

\subsection{Motivation of students}

Motivation of students is an important element to achieve high question/answer production. We proposed a simple game based on receiving reward [11]. Students get points for an action performed. Number of points varies according activity of the whole group of students. Our aim is to achieve a balance between creating and answering questions. The students see actual points and can compare with their peers. Their job is to find a tactic that brings them the greatest number of points (in principle, adding quality questions and rate questions like the others).

Motivation is also needed to create questions evenly across educational material. Therefore, we decided to motivate and benefit the students who add questions in such place of the whole set of learning objects where no or just few questions exist. Educational materials already including questions are presented in the navigational panel by an indicator. We use two types of indicators. One to indicate that sufficient number of questions is already added to a given learning object, and the second for an indication of just few questions. Learning objects without any questions are not marked. If the student adds a question to the place where it is just a few questions or none, he gets a higher score.

\section{Evaluation}

For evaluation of the proposed method we developed a software component, which is a part of adaptive learning framework ALEF framework (Fig. 1) [10]. The ALEF framework is based on our previous system FLIP [12]. It adds openness and flexibility, 


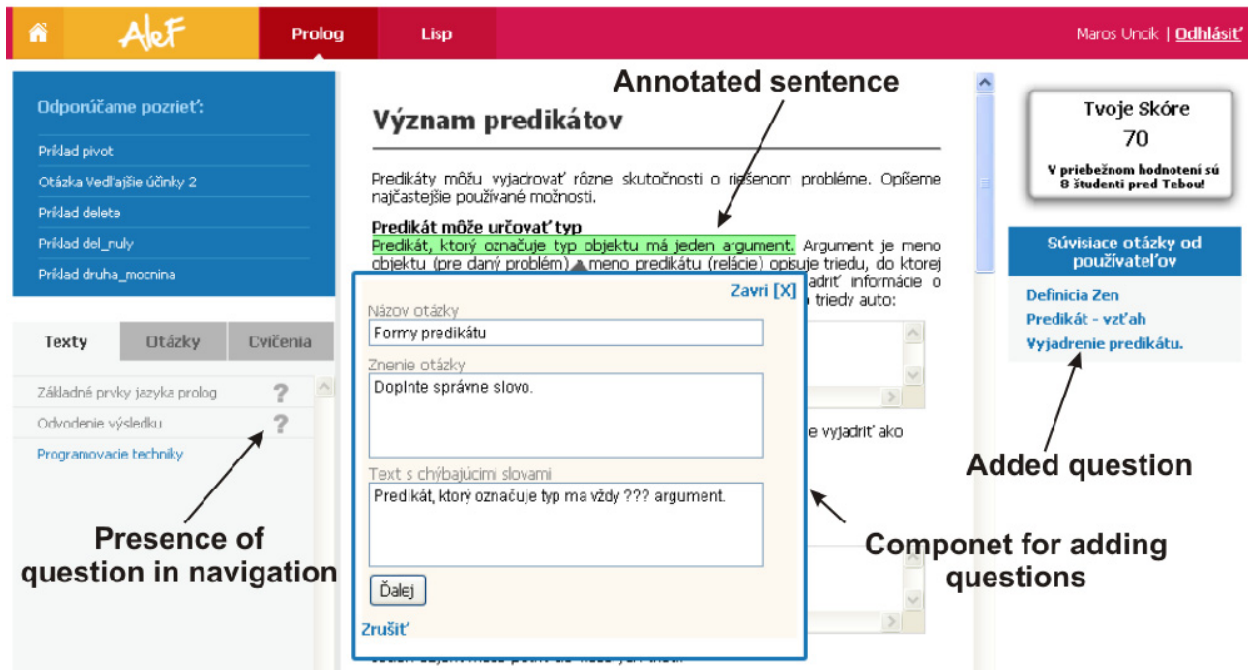

Fig.1. A screenshot of adaptive educational framework ALEF.

which allows development of data coupled software components within web-based infrastructure. Using the ALEF framework brings an advantage of full featured e-learning portal, which is already deployed in a real educational process (for several subjects of learning programming). The students had before our experiments already the opportunity to be acquainted with the system for a longer period of time.

ALEF offers the solution to adapt our method to the core data model containing data entities for annotations. We extended classic text annotation with a structure for questions of several types.

Students add interactive content, the questions, which will be answered by their peers. This implicates that we designed such types of questions, which answers can be evaluated automatically. In current implementation students may add four types of questions: single choice, multiple choice, sorter and text complement question.

We implemented the creating a question as a procedure similar to adding an annotation:

1. The student selects text to be enriched.

2. The student selects the type of the question.

3. The student fills the form for adding a question, generally specifies title, description and possible answers and indicates the correct answer.

4. The question is added to the educational text

(related to selected text and actual learning object).

This process results in a set of questions, which are rated by our method. The presence of the question bound to the educational text is highlighting with a gray or black question mark in the navigational panel of the ALEF screen.

We evaluated the method of question acquisition in the experiment aimed to an analysis of added questions by students. The experiment was conducted in real settings in the domain of logic programming. Students got educational materials for learning programming language Prolog (textbook of Functional and logic programming, which is used to teach this course at the Slovak University of Technology in Bratislava). Students created questions, and/or answered and rated existing questions.

After seven days since the start of the experiment, we provided the evaluation. The experiment involved 30 students (nearly $60 \%$ of all students that could participate). Students created together 88 questions and provided 660 answers and 33 bug reports.

We manually evaluated all questions created by the students and divided them into three levels according to their quality (Tab. 1). In one set we put questions, which contained an error or were ambiguous, so useless for educational purposes. On the other hand, we created the set containing questions which we consider to be of high quality, and challenging enough to be used within educational materials. We were guided by quality of questions that already exist in the system as a part of learning objects. Further set contains correct questions of medium quality, e.g. their difficulty is rather low or they are not spelled properly.

The same sample of questions was evaluated using proposed method. The method estimated 24 from all 88 as quality questions. We compared these questions with the questions evaluated manually.

Tab.1. Manual evaluation of questions.

\begin{tabular}{l|l}
\hline \multicolumn{2}{c}{ All questions } \\
\hline Set & No. of questions \\
\hline High quality questions & 33 \\
Average quality questions & 48 \\
Faulty questions & 7 \\
\hline
\end{tabular}


Tab. 2. Automatic evaluated questions.

\begin{tabular}{l|l}
\hline \multicolumn{2}{c}{24 questions marked as quality questions } \\
\hline Set & No. of questions \\
\hline High quality questions & 17 \\
Average quality questions & 7 \\
Faulty questions & 0 \\
\hline
\end{tabular}

According to Tab. 2, the question quality rating model did not estimate for quality questions only those of high quality, but also some questions with the lower level of quality. Important result is that none of the faulty questions was marked as quality one.

Another aspect is the level of knowledge and motivation of students who participated. We found out that $70 \%$ of those students who achieved the best results in the Functional and logic programming course (they were among $30 \%$ of top students) were active in our experiment and actually were evaluated by our student rating model among the top $30 \%$.

\section{Conclusions and future work}

The content enrichment by annotation is a useful approach for gaining new content or additional information. In educational context annotations present added value for learning objects and a useful assistance for students. We have presented an approach for gaining new and quality content by learners (students) in form of questions for a large volume of educational texts available for exploration in an educational system.

Our contribution mainly is in devising a new way of enriching hypermedia in educational domain with effects for direct improvement of learning by involving students into the learning process and facilitating a collaboration. Moreover, indirect effects include acquiring the sources for better adaptation using user ratings (e.g., to complement a user model based on concept ratings [9]), which well reflects actual user knowledge and the possibility of including high quality questions to learning objects, and use them even for exams as we did in Functional and Logic Programming course in this academic year. This not only simplified job of the teacher, but also made it more interesting as students were more involved.

In our future work we plan to explore further exploitation of the acquired questions for an analysis of the documents with aim to identify metadata (concepts) related to particular document (learning object). The other work embraces searching new forms of motivation for students. One option is to find new games and activities related to the acquisition of questions for students. Such action may be looking for duplicate questions. We believe that the collaborative acquisition of the questions has the great potential and we continue in its further research and improvement.

Acknowledgement. This work was supported by the Scientific Grant Agency of SR, grant No. VG1/0508/09, the Cultural and Educational Grant Agency of SR, grant No. 028-025STU-4/2010, and it is a partial result of the Research \& Development Operational Program for the project Support of Center of Excellence for Smart Technologies, Systems and Services II, ITMS 25240120029, co-funded by ERDF.

\section{References}

[1] Barla, M., Bieliková, M., Bou Ezzeddinne, A., Kramár, T., Šimko, M., Vozár, O.: On the Impact of Adaptive Test Question Selection for Learning Efficiency. In: Computers \& Education. Vol. 55, No. 2, 2010, 846-857.

[2] Bateman, S., Brooks, C., McCalla, G., and Brusilovsky, P.: Applying Collaborative Tagging to E-Learning. In: Proc. of Workshop on Tagging and Metadata for Social Information Organization at WWW 2007, 2007.

[3] Bonifazi, F., Levialdi, S., Rizzo, P., and Trinchese, R.: A web-based annotation tool supporting e-learning. In: Proc. of the Working Conf. on Advanced Visual interfaces, ACM, 2002, pp. 123-128.

[4] Farzan R., Brusilovsky P.: AnnotatEd: A Social Navigation and Annotation Service for Web-based Educational Resources, In: Proc of E-Learn'06, 2006, pp. 2794-2802.

[5] Hsiao, I. H., Brusilovsky, P.: Modeling Peer Review in Example Annotation. In: Proc. of 16th Int. Conf. on Computers in Education, 2008, pp. 357-362.

[6] Lawton, D. T., Smith, I. E.: The Knowledge Weasel hypermedia annotation system. In: Proc. of the 5th ACM Conf. on Hypertext, ACM, 1993, pp. 106-117.

[7] Mihál V., Bieliková M.: An Approach to Annotation of Learning Texts on Programming within a Web-Based Educational System. In: Proc. of SMAP'09, IEEE CS, 2009, pp. 99-104.

[8] Reeve, L., Han H.: Survey of semantic annotation platforms. In: Proc. of the 2005 ACM Symposium on Applied Computing, ACM, 2005, pp. 1634 - 1638.

[9] Šaloun, P., Velart, Z.: Concept Space Rating for Personalization of Learning Materials based on Relations. In: Proc. of SMAP'09, IEEE CS, 2009, pp. 67-72.

[10] Šimko M., Barla M. and Bieliková M.: ALEF: A Framework for Adaptive Web-based Learning 2.0. In: Proc. of World Computer Congress, KCKS 2010, IFIP AICT 324, 2010, pp. 367-378.

[11] Tüzün, H. et al.: The effects of computer games on primary school students' achievement and motivation in geography learning. In Computers \& Education, Vol. 59, No. 1, 2009, pp. 68-77.

[12] Vozár, O., Bieliková, M.: Adaptive Test Question Selection for Web-based Educational System. In: Proc. of 3rd Int. Workshop on Semantic Media Adaptation and Personalization, SMAP08. IEEE CS, 2008, pp. 164-169. 